\title{
Hamiltonian potential functions for differential games
}

\author{
Davide Dragone, ${ }^{\S}$ Luca Lambertini, ${ }^{\S}$ George Leitmann\# \\ and Arsen Palestini ${ }^{\S}$ \\ $\S$ Department of Economics, University of Bologna \\ Strada Maggiore 45, 40125 Bologna, Italy \\ davide.dragone@unibo.it, luca.lambertini@unibo.it, palestini@math.unifi.it \\ \# College of Engineering, University of California at Berkeley \\ Berkeley, CA 94720, USA; gleit@berkeley.edu
}

September 2, 2008 


\begin{abstract}
We introduce the concept of Hamiltonian potential function for noncooperative open-loop differential games with $n$ players, $n$ controls and $n$ states, and characterise a sufficient condition for its existence. We also identify a class of games admitting a Hamiltonian potential and provide appropriate examples pertaining to advertising, industrial organization and macroeconomic policy.
\end{abstract}

Keywords: differential games, potential games, open-loop equilibrium

JEL codes: C73 


\section{Introduction}

Following Monderer and Shapley (1996), a relatively large literature has investigated potential functions for static games. In a potential game, the information about Nash equilibria is nested into a single real-valued function (the potential function) over the strategy space. The specific feature of a potential function defined for a given game is that the gradient of the corresponding potential function coincides with the vector of first derivatives of the individual payoff functions of the original game itself. As stressed by Slade (1996), the interest of this line of research is that, in a game admitting a potential function it is as if players were jointly maximising that single function instead of competing to maximise their respective payoffs.

To the best of our knowledge, no attempt has been made as yet concerning the construction of a potential function for differential games. Here, we will confine our attention to the solution of noncooperative open-loop differential games. Given that the necessary conditions for the solution of an open-loop dynamic game contain the adjoint equations in addition to the first order conditions on controls, verifying the existence of a potential function for such a game is essentially different from carrying out the same task for a static game. What a potential function must accomplish in a dynamic game is to reproduce the same dynamic system (state and control equations) and achieve the same open-loop solution(s) the original game yields. We refer to this function as a Hamiltonian potential function.

We provide a sufficient condition for the existence of the Hamiltonian potential function in a generic noncooperative open-loop differential game, and subsequently identify

a class of games admitting a Hamiltonian potential function. Then, we illustrate three examples belonging to different areas of economics and management. 


\section{A potential for differential games}

\subsection{Potential in static games}

We briefly recall the concept of potential in static noncooperative, full information games. We borrow from physics the following:

Definition 1 A given vector field $F=\left(F_{1}(s), \ldots, F_{n}(s)\right)$ is conservative if there exists a differentiable function $P(s)$ such that:

$$
\frac{\partial P(s)}{\partial s_{i}}=F_{i}(s), \quad i=1, \ldots, n .
$$

$P(s)$ is called a potential function for $F$.

Given a game $G$ where $N=\{1,2,3, \ldots, n\}$ is the set of players, each one of them endowed with the profit function $\pi_{i}(\cdot)$, if the vector field $\left(\pi_{1}(\cdot), \ldots, \pi_{n}(\cdot)\right)$ is conservative, then it admits a potential function and it is an exact potential game in the sense of Monderer and Shapley (1996).

A potential in a static framework contains all the relevant information of the original static $n$-players game. Analogously, a Hamiltonian potential contains all the relevant information of the original dynamic $n$-players game. In next subsection we consider a class of differential games and we provide some requirements allowing for the construction of a Hamiltonian potential. Note that this definition will necessarily differ from the one given in Monderer and Shapley (1996) and the rest of the related literature, as state variables do not appear in static games.

\subsection{The dynamic set up: definitions}

In the following, we define a (normal) differential game, together with the appropriate requirements allowing for the construction of a Hamiltonian potential function, if it exists.

Consider an infinite horizon differential game $\Gamma$ with the following features:

- $n$ is the number of players; 
- $\mathbf{x}(t)=\left(x_{1}(t), \ldots, x_{n}(t)\right) \in X \subset \mathbb{R}^{n}$, where $X$ is a bounded and open set, is a vector of state variables;

- $\mathbf{u}(t) \in U:=U_{1} \times \ldots \times U_{n}$ is a vector of control variables; $u_{i}(t)$ is the control related to the $i$-th player. The set $U_{i}$ is also bounded and open for every $i=1, \ldots, n$;

- the $i$-th player is endowed with the instantaneous payoff $\pi_{i}(\mathbf{x}(t), \mathbf{u}(t), t)$ and is supposed to maximize the discounted objective functional:

$$
J_{i} \equiv \int_{t_{0}}^{\infty} e^{-\rho t} \pi_{i}(\mathbf{x}(t), \mathbf{u}(t), t) d t
$$

subject to the kinematic equation:

$$
\left\{\begin{array}{l}
\dot{x}_{i}(t)=g_{i}(\mathbf{x}(t), \mathbf{u}(t), t) \\
x_{i}\left(t_{0}\right)=x_{i 0}
\end{array},\right.
$$

where $g_{i}(\cdot) \in C^{2}\left(X \times U \times\left[t_{0}, \infty\right)\right), i=1, \ldots, n$ and $\rho$ is the intertemporal discount rate, constant and common to all agents. ${ }^{1}$

That is, we confine our attention to a setup with $n$ players, each endowed with one control and one state. This is of course a special case; however, it is of interest in terms of applications to economics, where this structure is rather common.

The standard technique requires constructing a Hamiltonian function of each agent as follows:

$$
H_{i}(\cdot)=\pi_{i}(\mathbf{x}(t), \mathbf{u}(t), t)+\lambda_{i i}(t) g_{i}(\mathbf{x}(t), \mathbf{u}(t), t)+\sum_{j \neq i} \lambda_{i j}(t) g_{j}(\mathbf{x}(t), \mathbf{u}(t), t),
$$

where $\lambda_{i j}(t)$ is the costate variable associated by player $i$ with state variable $x_{j}$; suppose $H_{i} \in C^{2}\left(X \times U \times \mathbb{R}^{n \times n} \times\left[t_{0}, \infty\right)\right) . x(0)$ is the vector of initial conditions on states.

Here we confine our attention to interior open-loop Nash (i.e., simultaneous) equilibria. The related necessary conditions taken on (3) are (omitting arguments for brevity):

$$
\frac{\partial H_{i}}{\partial u_{i}}=0 \Leftrightarrow \frac{\partial \pi_{i}}{\partial u_{i}}+\lambda_{i i} \frac{\partial g_{i}}{\partial u_{i}}+\sum_{j \neq i} \lambda_{i j} \frac{\partial g_{j}}{\partial u_{i}}=0
$$

\footnotetext{
${ }^{1}$ For the sake of simplicity, we assume that all agents have the same time preferences.
} 


$$
\begin{gathered}
-\frac{\partial H_{i}}{\partial x_{i}}=\dot{\lambda}_{i i}-\rho \lambda_{i i} \\
-\frac{\partial H_{i}}{\partial x_{j}}=\dot{\lambda}_{i j}-\rho \lambda_{i j} \forall j \neq i
\end{gathered}
$$

plus the transversality conditions:

$$
\lim _{t \rightarrow \infty} \lambda_{i j}=0 \forall i, j .
$$

In a static game, the construction of the corresponding potential function (if it exists) requires integrating the $n$ first-order conditions on choice variables, summing up the integrals and checking whether what results from this procedure is indeed a conservative field.

In a differential game framework, we are looking for the potential function of a game with $n$ controls and $n$ states, and therefore also $n$ costates. The whole set of necessary conditions taken for the population of players consists therefore of $n$ FOCs on controls (like (4), one for each player) and $n \times n$ costate equations (like (5-6), $n$ for each player). Now, there immediately arises a difficulty with costates, as (4) (and, most likely, (5)), will contain $\lambda_{i j}$ (for at least some $j \neq i$ ). Therefore, integrating all partial derivatives $\partial H_{i} / \partial x_{j}, j \neq i$, and summing them up, would yield a function containing not $n$ but $n \times n$ costates. Clearly, if it can be established that either $\lambda_{i j}=0$ for all $j \neq i$, or costates $\lambda_{i j}$ do not appear in (4-5), then, taking into account FOCs (4), the set of partial derivatives to be integrated reduces to $2 n$ and the game may admit a potential function. These properties can be checked either by examination of the necessary conditions or when one of the following cases occurs.

- Consider the most general case of all, with $\dot{x}_{i}=g_{i}(\mathbf{x}, \mathbf{u}, t)$ for all $i=1, \ldots, n$. If so, then

$$
\frac{\partial^{2} H_{i}}{\partial u_{i} \partial \lambda_{i j}} \neq 0 \text { and } \frac{\partial^{2} H_{i}}{\partial x_{i} \partial \lambda_{i j}} \neq 0 .
$$

Therefore, we need (6) to admit the solution $\lambda_{i j}=0$ at all $t$.

- A simpler case is that where $\dot{x}_{i}=g_{i}\left(\mathbf{x}, u_{i}, t\right)$ for all $i=1, \ldots, n$. Here,

$$
\frac{\partial^{2} H_{i}}{\partial u_{i} \partial \lambda_{i j}}=0 \text { but } \frac{\partial^{2} H_{i}}{\partial x_{i} \partial \lambda_{i j}} \neq 0,
$$


and again we need (6) to admit the solution $\lambda_{i j}=0$ at all $t$.

- The third case is that where $\dot{x}_{i}=g_{i}\left(x_{i}, \mathbf{u}, t\right)$ for all $i=1, \ldots, n$. also here we need (6) to admit the solution $\lambda_{i j}=0$ at all $t$, since

$$
\frac{\partial^{2} H_{i}}{\partial u_{i} \partial \lambda_{i j}} \neq 0 \text { and } \frac{\partial^{2} H_{i}}{\partial x_{i} \partial \lambda_{i j}}=0 \text {. }
$$

- The last, and simplest, case is the setup where $\dot{x}_{i}=g_{i}\left(x_{i}, u_{i}, t\right)$ for all $i=1, \ldots, n$. As the game exhibits separate state dynamics,

$$
\frac{\partial^{2} H_{i}}{\partial u_{i} \partial \lambda_{i j}}=0 \text { and } \frac{\partial^{2} H_{i}}{\partial x_{i} \partial \lambda_{i j}}=0
$$

and therefore the solution of (6) w.r.t. $\lambda_{i j}$ is in fact irrelevant. ${ }^{2}$

Whenever the game does not feature separate state dynamics, a sufficient condition for (6) to admit the null solution is the following:

Proposition 2 If there exist $n-1$ functions $\varpi_{j}\left(x_{j}\right)$ such that

$$
\frac{\partial H_{i}}{\partial x_{j}}=\lambda_{i j} \varpi_{j}\left(x_{j}\right)
$$

for all $i, j=1, \ldots, n, i \neq j$, then (6) admits the solution $\lambda_{i j}=0$ at all $t$, for all $j \neq i$.

Proof. We rewrite the adjoint equations (6) in this case:

$$
\dot{\lambda}_{i j}-\rho \lambda_{i j}=-\lambda_{i j} \varpi_{j}\left(x_{j}\right),
$$

$\forall j \neq i$. This equations can be elementarily solved by separation of variables, and the solutions are:

$$
\lambda_{i j}(t)=\lambda_{i j}\left(t_{0}\right) e^{\int_{t_{0}}^{\infty}\left(\rho-\varphi_{j}\left(x_{j}(s)\right)\right) d s},
$$

obviously null for the initial condition $\lambda_{i j}\left(t_{0}\right)=0$.

In the remainder, we confine our attention on games where either (i) state dynamics are separate, or (ii) any adjoint equation (6) admits the solution $\lambda_{i j}=0$ at all $t$. It is now convenient to define vector $\boldsymbol{\lambda}_{i i}:=\left(\lambda_{11}, \lambda_{22}, \ldots, \lambda_{j j}, \ldots, \lambda_{n n}\right)$.

\footnotetext{
${ }^{2}$ Observe that, if $\lambda_{i j}$ is either nil or irrelevant, the symmetry assumption concerning time discounting is not critical.
} 
Definition 3 Given a differential game $\Gamma$ with Hamiltonians $H_{i}$, we define the Hamiltonian potential for game $\Gamma$ as the function $H_{P}\left(\mathbf{u}, \mathbf{x}, \boldsymbol{\lambda}_{i i}\right)$, such that:

$$
\left(\frac{\partial H_{P}}{\partial u_{1}}, \ldots, \frac{\partial H_{P}}{\partial u_{n}}, \frac{\partial H_{P}}{\partial x_{1}}, \ldots, \frac{\partial H_{P}}{\partial x_{n}}\right)=\left(\frac{\partial H_{1}}{\partial u_{1}}, \ldots, \frac{\partial H_{n}}{\partial u_{n}}, \frac{\partial H_{1}}{\partial x_{1}}, \ldots, \frac{\partial H_{n}}{\partial x_{n}}\right) .
$$

If it exists, a Hamiltonian potential function $H_{P}$ for game $\Gamma$ is a real-valued function that contains all the relevant information of the original differential game. The requirement on $H_{P}$ is that the partial derivatives of the individual Hamiltonian functions with respect to states and controls are replicated, i.e., that the gradient of $H_{P}$ w.r.t. states $x_{i}(t)$ and controls $u_{i}(t)$ replicates $\partial H_{i}(\cdot) / \partial x_{i}(t)$ and $\partial H_{i}(\cdot) / \partial u_{i}(t)$ for all $i$.

\subsection{Hamiltonian potential: construction and properties}

The Hamiltonian potential is the Hamiltonian function of a fictitious player replacing the original $n$ players involved in game $\Gamma$, and endowed with the task of replicating the set of FOCs on controls as well as the $n$ adjoint equations that are neither irrelevant nor admit a null solution, and finally reproducing the same control dynamics as in the original game. The final outcome of this procedure must be the same dynamic system of state and control equations as in game $\Gamma$.

Now we show the construction of a Hamiltonian potential and a class of differential games that admit a Hamiltonian potential.

Proposition 4 If there exists a function $\widehat{P}\left(u_{1}, \ldots, u_{n}, x_{1}, \ldots, x_{n}, \boldsymbol{\lambda}_{i i}\right)$ such that:

$$
\begin{aligned}
& \frac{\partial \widehat{P}}{\partial u_{i}}+\lambda_{i i} \frac{\partial \dot{x}_{i}}{\partial u_{i}}+\sum_{j \neq i} \lambda_{j j} \frac{\partial \dot{x}_{j}}{\partial u_{i}}=\frac{\partial H_{i}}{\partial u_{i}} \\
& \frac{\partial \widehat{P}}{\partial x_{i}}+\lambda_{i i} \frac{\partial \dot{x}_{i}}{\partial x_{i}}+\sum_{j \neq i} \lambda_{j j} \frac{\partial \dot{x}_{j}}{\partial x_{i}}=\frac{\partial H_{i}}{\partial x_{i}}
\end{aligned}
$$

for all $i, j=1, \ldots, n$, then the game $\Gamma$ with Hamiltonians $H_{i}$ admits a Hamiltonian potential with the following form:

$$
H_{P}\left(\mathbf{u}, \mathbf{x}, \boldsymbol{\lambda}_{i i}\right)=\widehat{P}\left(u_{1}, \ldots, u_{n}, x_{1}, \ldots, x_{n}, \boldsymbol{\lambda}_{i i}\right)+\sum_{i=1}^{n} \lambda_{i i} \dot{x}_{i} .
$$


Proof. It immediately follows from Definition 2.

Relying on the required structure, we can set up a class of differential games admitting a Hamilton potential and subsequently state a formula for such a function. As a preliminary step in this direction, we introduce the following:

Definition 5 A Hamiltonian of the type:

$$
H_{i}\left(\mathbf{u}, \mathbf{x}, \boldsymbol{\lambda}_{i i}\right)=\alpha_{i}\left(u_{i}, \boldsymbol{\lambda}_{i i}\right)+\sum_{j \neq i} \alpha_{j}\left(u_{j}, \boldsymbol{\lambda}_{i i}\right)+\gamma_{i}\left(x_{i}, \boldsymbol{\lambda}_{i i}\right)+\sum_{j \neq i} \gamma_{j}\left(x_{j}, \boldsymbol{\lambda}_{i i}\right)
$$

where $\alpha_{i}(\cdot)$ and $\gamma_{i}(\cdot)$ are $C^{2}$ functions with respect to all their variables, for all $i=1, \ldots, n$, is called additively separable in states and controls.

Proposition 6 Every differential game $\Gamma$ with additively separable Hamiltonians as in Definition 5 admits a Hamiltonian potential.

Proof. The additively separable form of the Hamiltonian functions allows us to obtain a conservative vector field. In fact, consider the $2 n$ coordinate vector

$$
\left(\frac{\partial \alpha_{1}}{\partial u_{1}}, \ldots, \frac{\partial \alpha_{n}}{\partial u_{n}}, \frac{\partial \gamma_{1}}{\partial x_{1}}, \ldots, \frac{\partial \gamma_{n}}{\partial x_{n}}\right)
$$

The $j$-th component of this vector is a function which only depends on the costate variables and on either the $j$-th control or the $(j-n)$-th state, so that a potential for this vector field exists and it is easily calculable.

It is worth stressing that the regularity requirements on the functions appearing in (18) might be considered much too restrictive, but in several economic models they are certainly satisfied. Appropriate examples will be presented in the next section.

When Proposition 6 holds, it may be useful to reformulate the FOCs and the adjoint equations of the differential game to achieve the open-loop equilibrium trajectories:

$$
\begin{gathered}
\frac{\partial H_{P}}{\partial u_{i}}=0 \Longleftrightarrow \frac{\partial \alpha_{i}}{\partial u_{i}}=0 \\
\dot{\lambda}_{i i}-\rho \lambda_{i i}=-\frac{\partial H_{P}}{\partial x_{i}}=-\frac{\partial \gamma_{i}}{\partial x_{i}}
\end{gathered}
$$


plus the appropriate transversality conditions.

By differentiating the FOCs with respect to time and by substitution in (20), we obtain a set of first order differential equations. Together with the equations of motion of the state variables, the dynamic system in states and controls completely describes the optimal trajectories of the game.

In the remainder, we illustrate two versions of the Leitmann and Schmitendorf (1978) differential game of advertising, a macroeconomic policy game and an advertising game with product quality dynamics. The first three games are characterised by null solutions to (6), while the fourth model is an example of the case where every $\lambda_{i j}$, for all $j \neq i$, is irrelevant.

\section{Examples}

\subsection{The original Leitmann-Schmitendorf differential game}

Consider now the game proposed by Leitmann and Schmitendorf $(1978),{ }^{3}$ where 2 firms sell substitutable goods and invest in advertising to determine their market share $x_{i} \geq 0$, with $i \in\{1,2\}$. Individual market share evolves over time as follows:

$$
\begin{aligned}
& \dot{x}_{1}=u_{1}-b_{1} x_{1}-\frac{c_{1} u_{1}^{2}}{2}-a_{1} x_{1} u_{2}, \\
& \dot{x}_{2}=u_{2}-b_{2} x_{2}-\frac{c_{2} u_{2}^{2}}{2}-a_{2} x_{2} u_{1},
\end{aligned}
$$

where $u_{i} \in\left[0,1 / c_{i}\right)$ is firm $i$ 's advertising control, ${ }^{4} a_{i}, b_{i}$ and $c_{i}$ are positive parameters, and shares are subject to the following constraints:

$$
x_{1}(t), x_{2}(t) \geq 0, x_{1}(t)+x_{2}(t) \leq 1
$$

Investment in advertising has a linear cost, so that the profit function for player $i$ is

\footnotetext{
${ }^{3}$ See also Feichtinger (1983).

${ }^{4}$ In the original version, the set of controls is compact. However, here we assume it is bounded and open and confine our attention to interior solutions. This has no particular bearings on the (non) existence of the Hamiltonian potential.
} 


$$
\pi_{i}=p_{i} x_{i}-u_{i}
$$

The problem is

$$
\max _{u_{i}} J_{i} \equiv \int_{0}^{\infty} e^{-\rho t} \pi_{i} d t
$$

subject to (21) and (22). The corresponding current-value Hamiltonian functions for player 1 and 2 are, respectively (we omit the dependence on the costate variables):

$$
\begin{aligned}
H_{1}\left(u_{1}, u_{2}, x_{1}, x_{2}\right)= & \pi_{1}+\lambda_{11} \dot{x}_{1}+\lambda_{12} \dot{x}_{2} \\
= & p_{1} x_{1}-u_{1}+\lambda_{11}\left(u_{1}-b_{1} x_{1}-\frac{c_{1} u_{1}^{2}}{2}-a_{1} x_{1} u_{2}\right) \\
& +\lambda_{12}\left(u_{2}-b_{2} x_{2}-\frac{c_{21} u_{2}^{2}}{2}-a_{2} x_{2} u_{1}\right) \\
H_{2}\left(u_{1}, u_{2}, x_{1}, x_{2}\right)= & \pi_{2}+\lambda_{21} \dot{x}_{1}+\lambda_{22} \dot{x}_{2} \\
= & p_{1} x_{2}-u_{2}+\lambda_{21}\left(u_{1}-b_{1} x_{1}-\frac{c_{1} u_{1}^{2}}{2}-a_{1} x_{1} u_{2}\right) \\
& +\lambda_{22}\left(u_{2}-b_{2} x_{2}-\frac{c_{21} u_{2}^{2}}{2}-a_{2} x_{2} u_{1}\right)
\end{aligned}
$$

The necessary conditions are:

$$
\begin{gathered}
\frac{\partial H_{i}}{\partial u_{i}}=0 \Leftrightarrow-1+\lambda_{i i}\left(1-c_{i} u_{i}\right)-\lambda_{i j} a_{j} x_{j}=0 \\
-\frac{\partial H_{i}}{\partial x_{i}}=\dot{\lambda}_{i i}-\rho \lambda_{i i} \Leftrightarrow \dot{\lambda}_{i i}=\lambda_{i i}\left(b_{i}+a_{i} u_{j}+\rho\right)-p_{i} \\
-\frac{\partial H_{i}}{\partial x_{j}}=\dot{\lambda}_{i j}-\rho \lambda_{i j} \Leftrightarrow \dot{\lambda}_{i j}=\lambda_{i j}\left(b_{j}+a_{j} u_{i}+\rho\right)
\end{gathered}
$$

with $j=-i$. Given that the game is state redundant and (25) admits the solution $\lambda_{i j}=0$ at all $t$, this costate equation and the related partial derivative $\partial H_{i} / \partial x_{j}$ can be disregarded. If we try to construct the potential for this game integrating the partial derivatives of all Hamiltonians with respect to the four variables (two states and two controls) we obtain the following:

$$
\Psi=\sum_{i=1}^{2}\left(\int \frac{\partial H_{i}}{\partial x_{i}} d x_{i}+\int \frac{\partial H_{i}}{\partial u_{i}} d u_{i}\right)=\sum_{i=1}^{2}\left[p_{i} x_{i}+u_{i}\left(\lambda_{i i}-1-a_{j} \lambda_{j j} x_{j}\right)\right]+
$$




$$
-\sum_{i=1}^{2}\left(b_{i} \lambda_{i i} x_{i}+\frac{1}{2} c_{i} \lambda_{i i} u_{i}^{2}\right)
$$

with

$$
\begin{gathered}
\frac{\partial \Psi}{\partial u_{i}}=-1+\lambda_{i i}\left(1-c_{i} u_{i}\right)-\lambda_{j j} a_{j} x_{j} \\
\frac{\partial \Psi}{\partial x_{i}}=p_{i}-b_{i} \lambda_{i i}-\lambda_{i i} a_{i} u_{j} .
\end{gathered}
$$

Clearly, (28) coincides with $\partial H_{i} / \partial x_{i}$, while (27) differs from $\partial H_{i} / \partial u_{i}$, which, taking into account $\lambda_{i j}=0$, rewrites as $-1+\lambda_{i i}\left(1-c_{i} u_{i}\right)$, whereby

$$
\frac{\partial \Psi}{\partial u_{i}}-\frac{\partial H_{i}}{\partial u_{i}}=-\lambda_{j j} a_{j} x_{j}
$$

which is the external effect exerted on the state of firm $j$ via the control $u_{i}$. Now, if one tries to clean this effect away, using

$$
\widehat{\Psi}=\Psi+\sum_{i=1}^{2} \lambda_{i i} a_{i} x_{i} u_{j}
$$

the result is

$$
\begin{gathered}
\frac{\partial \widehat{\Psi}}{\partial u_{i}}=-1+\lambda_{i i}\left(1-c_{i} u_{i}\right) \\
\frac{\partial \widehat{\Psi}}{\partial x_{i}}=p_{i}-b_{i} \lambda_{i i}
\end{gathered}
$$

so that the partial derivatives taken on controls now coincide with the corresponding FOCs of the original game, but this is no longer true for the partial derivatives w.r.t. states. Hence, the associated vector field is not conservative and the game does not admit a potential function.

It is worth stressing that this is the consequence of the spillover (or external) effects appearing in the state equations, which are designed in such a way that they introduce a problematic interplay between the state of player $i$ and the control of player $j$. The next section illustrates a different version of the advertising game, where this aspect is accounted for without compromising the structure and properties of the original game, and, at the same time, to ensure the existence of a potential function. 


\subsection{A simplified version}

Consider now a simplified version of the previous game where the individual market share changes over time as a function of the investment in advertising $u_{i} \geq 0$ of each firm and decreases over time at a fixed rate $\delta \geq 0$ :

$$
\dot{x}_{i}=u_{i}+s u_{j}-\delta x_{i} .
$$

Note that here (31) still features an externality from the control of $j$ onto the state of $i$ but there is no direct interplay between $u_{j}$ and $x_{i}$; parameter $s \in[-1,1]$ measures the spillover effect, as in the static version. The instantaneous profit of firm $i$ is $\pi_{i}=p x_{i}-b u_{i}^{2}{ }^{5}$ Denoting by $\rho \geq 0$ the intertemporal discount rate, each firm has to determine the amount of advertising that solves the following problem

$$
\max _{u_{i}} J_{i} \equiv \int_{0}^{\infty} e^{-\rho t} \pi_{i} d t
$$

subject to (31). The corresponding current-value Hamiltonians are:

$$
\begin{aligned}
& H_{1}(\cdot)=\pi_{1}+\lambda_{11} \dot{x}_{1}+\lambda_{12} \dot{x}_{2} \\
& H_{2}(\cdot)=\pi_{2}+\lambda_{22} \dot{x}_{2}+\lambda_{21} \dot{x}_{1}
\end{aligned}
$$

The application of Pontryiagin's Maximum Principle leads to the following necessary optimality conditions:

$$
\begin{gathered}
\frac{\partial H_{1}}{\partial u_{1}}=-2 b u_{1}+\lambda_{11}+\lambda_{12} s \\
\frac{\partial H_{2}}{\partial u_{2}}=-2 b u_{2}+\lambda_{22}+\lambda_{21} s \\
\dot{\lambda}_{11}=\rho \lambda_{11}-\frac{\partial H_{1}}{\partial x_{1}}=(\rho+\delta) \lambda_{11}+p \\
\dot{\lambda}_{12}=\rho \lambda_{12}-\frac{\partial H_{1}}{\partial x_{2}}=(\rho+\delta) \lambda_{12}
\end{gathered}
$$

\footnotetext{
${ }^{5}$ For simplicity we set $p_{i}=p_{j}=p$, involving no loss of generality.
} 


$$
\begin{gathered}
\dot{\lambda}_{22}=\rho \lambda_{22}-\frac{\partial H_{2}}{\partial x_{2}}=(\rho+\delta) \lambda_{22}+p \\
\dot{\lambda}_{21}=\rho \lambda_{21}-\frac{\partial H_{2}}{\partial x_{1}}=(\rho+\delta) \lambda_{21}
\end{gathered}
$$

where, since Proposition 2 applies, the solutions $\lambda_{12} \equiv 0, \lambda_{21} \equiv 0$ are admissible at every instant, implying $\partial H_{1} / \partial x_{2}=\partial H_{2} / \partial x_{1}=0$ at all $t$. On the basis of conditions (32-35), we can state:

Proposition 7 The game is state redundant. Therefore, the open-loop equilibrium is a degenerate feedback (i.e., it is strongly time consistent).

Differentiating (32) with respect to time yields

$$
\begin{aligned}
& \dot{u}_{1}=\frac{1}{2 b}\left(\dot{\lambda}_{11}+\dot{\lambda}_{12} s\right)=u_{1}(\delta+\rho)-\frac{p}{2 b} \\
& \dot{u}_{2}=\frac{1}{2 b}\left(\dot{\lambda}_{22}+\dot{\lambda}_{21} s\right)=u_{2}(\delta+\rho)-\frac{p}{2 b}
\end{aligned}
$$

The steady state values of $u_{i}^{s s}$ and $x_{i}^{s s}$ are:

$$
\begin{aligned}
& u_{1}^{s s}=u_{2}^{s s}=\frac{p}{2 b(\delta+\rho)} \\
& x_{1}^{s s}=x_{2}^{s s}=\frac{p}{2 b \delta(\delta+\rho)}(1+s)
\end{aligned}
$$

which yield the steady state individual profits:

$$
\pi_{1}^{s s}=\pi_{2}^{s s}=\frac{p^{2}}{4 b \delta(\delta+\rho)^{2}}[\delta+2 \rho+2(\delta+\rho) s] .
$$

Note that, in the limit case in which there is no baseline demand, the investment in advertisement completely depreciates overnight and the firm does not discount future payoffs, i.e. $\bar{x}=0, \delta=1$ and $\rho=0$, the solution of the static and the differential game coincide. 
Since the Hamiltonian of the game is additively separable in states and controls, Proposition 6 applies and the following Hamiltonian potential $H_{P}(\cdot)$ can be constructed:

$$
\begin{aligned}
H_{P}\left(\mathbf{u}, \mathbf{x}, \boldsymbol{\lambda}_{i i}\right) & =\sum_{i=1}^{2}\left(\int \frac{\partial H_{i}}{\partial x_{i}} d x_{i}+\int \frac{\partial H_{i}}{\partial u_{i}} d u_{i}\right) \\
& =-b\left(u_{1}^{2}+u_{2}^{2}\right)+p\left(x_{1}+x_{2}\right)+\lambda_{11} u_{1}+\lambda_{22} u_{2}-\delta\left(\lambda_{11} x_{1}+\lambda_{22} x_{2}\right) \\
& =\widehat{P}(\cdot)+\lambda_{11}\left(u_{1}+s u_{2}-\delta x_{1}\right)+\lambda_{22}\left(u_{2}+s u_{1}-\delta x_{2}\right)
\end{aligned}
$$

where

$$
\widehat{P}\left(u_{1}, u_{2}, x_{1}, x_{2}, \lambda_{11}, \lambda_{22}\right)=-b\left(u_{1}^{2}+u_{2}^{2}\right)+p\left(x_{1}+x_{2}\right)-\left(\lambda_{11} u_{2}+\lambda_{22} u_{1}\right) s
$$

Therefore,

Proposition 8 The open-loop game admits a Hamiltonian potential.

That is, reshaping the externalities appearing in the state equations in such a way that they do not feature a multiplicative effect between states and controls yields as a result that the game is indeed a potential one. Note that the different approaches characterising the original Leitmann-Schmitendorf game and this modified version reflect two different definitions of the spillover effect that have been "coded" relatively long ago in the literature on R\&D for process innovation. The one appearing in Leitmann-Schmitendorf nests into what Kamien and Zang (2000) call absorptive capacity (because the ability of firm $i$ to affect the state of firm $j$ depends on the stock of firm $j$ 's state), while that appearing in the new version is independent of the state and replicates the functional form adopted by d'Aspremont and Jacquemin (1988) in a static model and Cellini and Lambertini (2005) in a dynamic one.

\subsection{A macroeconomic policy game}

The setup is a dynamic version of Lambertini and Rovelli (2004). The game takes place in a single country, between a central bank $(B)$ controlling the nominal interest rate $r \geq 0$ and a fiscal authority $(F)$ controlling the budget deficit $f \in(-\bar{f}, \bar{f})$. Where $\bar{f}$ is 
introduced to measure an exogenous upper (lower) bound to surplus (deficit). The states are the inflation rate $\pi$ and GNP (gross national product) $y$, whose respective dynamic equations are:

$$
\begin{aligned}
& \dot{\pi}=\beta f+\delta(r-\pi) \\
& \dot{y}=\varphi\left(y-y^{*}\right)+\eta f
\end{aligned}
$$

where $\beta, \delta, \eta, \varphi$ are positive parameters and $y^{*}$ is the benchmark (or full employment) level of income, exogenously given.

The instantaneous payoff functions of players are represented by the following loss functions:

$$
\begin{gathered}
L_{B}=\left(\pi-\pi^{*}\right)^{2}+\theta\left(r-r^{*}\right)^{2} \\
L_{F}=\left(y-y^{*}\right)^{2}+v f^{2}
\end{gathered}
$$

where $\theta$ and $v$ are positive parameters. As usual in this literature, the central banker is keen on using monetary policy to affect the behaviour of national income, the more so the higher is parameter $\theta$.

The Hamiltonian functions are:

$$
\begin{gathered}
H_{B}=\left(\pi-\pi^{*}\right)^{2}+\theta\left(r-r^{*}\right)^{2}+\lambda_{B \pi}[\beta f+\delta(r-\pi)]+\lambda_{B y}\left[\varphi\left(y-y^{*}\right)+\eta f\right] \\
H_{F}=\left(y-y^{*}\right)^{2}+v f^{2}+\lambda_{F \pi}[\beta f+\delta(r-\pi)]+\lambda_{F y}\left[\varphi\left(y-y^{*}\right)+\eta f\right] .
\end{gathered}
$$

The set of necessary conditions is:

$$
\begin{gathered}
\frac{\partial H_{B}}{\partial r}=2 \theta\left(r-r^{*}\right)+\delta \lambda_{B \pi}=0 \\
\frac{\partial H_{F}}{\partial f}=2 v f+\beta \lambda_{F \pi}+\eta \lambda_{F y}=0 \\
\dot{\lambda}_{B \pi}=(\rho+\delta) \lambda_{B \pi}-2\left(\pi-\pi^{*}\right) \\
\dot{\lambda}_{B y}=(\rho-\varphi) \lambda_{B y} \\
\dot{\lambda}_{F \pi}=(\rho+\delta) \lambda_{F \pi}
\end{gathered}
$$




$$
\dot{\lambda}_{F y}=(\rho-\varphi) \lambda_{F y}-2\left(y-y^{*}\right)
$$

Clearly, on the basis of Proposition 2 costate equations (48-49) admit the solution $\lambda_{B y}=$ $\lambda_{F \pi}=0$ at all $t$. Since Propositions 3 and 5 apply, we show the Hamiltonian potential of the present game:

$$
\begin{gathered}
H_{P}\left(\mathbf{u}, \mathbf{x}, \boldsymbol{\lambda}_{i i}\right)=\int \frac{\partial H_{B}}{\partial r} d r+\int \frac{\partial H_{B}}{\partial \pi} d \pi+\int \frac{\partial H_{F}}{\partial f} d f+\int \frac{\partial H_{F}}{\partial y} d y \\
=\theta\left(r-r^{*}\right)^{2}+\left(\pi-\pi^{*}\right)^{2}+v f^{2}+\left(y-y^{*}\right)^{2}+\lambda_{F y}(\eta f+\varphi y)+\lambda_{B \pi}(r-\pi) \delta \\
=\widehat{P}(\cdot)+\lambda_{B \pi} \dot{\pi}+\lambda_{F y} \dot{y}
\end{gathered}
$$

with

$$
\widehat{P}(\cdot)=\theta\left(r-r^{*}\right)^{2}+\left(\pi-\pi^{*}\right)^{2}+v f^{2}+\left(y-y^{*}\right)^{2}-\lambda_{B \pi} \beta f+\lambda_{F y} \varphi y^{*} .
$$

\subsection{A marketing game with product quality improvement}

Here we propose a simple marketing game where two firms invest in $R \& D$ to increase their respective product qualities, which in turn determine market shares. This game nests into a wide literature in marketing and management (see Feichtinger et al., 1994, and Jørgensen and Zaccour, 2004, for exhaustive surveys). Each firm $i$ sells a single good of quality $q_{i}, i \in\{H, L\}$, with $Q>q_{H} \geq q_{L} \geq 0, Q$ being the exogenously given utmost quality level which the industry can supply. At every instant, the market share of firm $i$ is $\sigma_{i}=\alpha+q_{i}-\xi q_{j}, \xi \in[0,1]$, and $\sigma_{i}+\sigma_{j} \leq 1$.

Qualities are state variables evolving according to the dynamics:

$$
\dot{q}_{i}=k_{i}-\varsigma q_{i}
$$

where $k_{i} \geq 0$ is the $\mathrm{R} \& \mathrm{D}$ control and $\varsigma>0$ is a constant decay rate common to both firms. The instantaneous $\mathrm{R} \& \mathrm{D}$ cost is $C_{i}\left(k_{i}\right)=c k_{i}^{2}$, and the instantaneous profit of firm $i$ is $\pi_{i}=p_{i} \sigma_{i}-c k_{i}^{2}$. We consider the price $p_{i}$ as an exogenously given parameter.

The resulting Hamiltonian of firm $i$ is:

$$
H_{i}=p_{i}\left[\alpha+q_{i}-\xi q_{j}\right]-c k_{i}^{2}+\lambda_{i i}\left(k_{i}-\varsigma q_{i}\right)+\lambda_{i j}\left(k_{j}-\varsigma q_{j}\right) .
$$


The first order condition on control is

$$
\frac{\partial H_{i}}{\partial k_{i}}=-2 c k_{i}+\lambda_{i i}=0
$$

and the adjoint equations are

$$
\begin{gathered}
\dot{\lambda}_{i i}=\lambda_{i i}(\rho+\varsigma)-p_{i} \\
\dot{\lambda}_{i j}=\lambda_{i j}(\rho+\varsigma)+\xi p_{i}
\end{gathered}
$$

Now observe that the differential equation describing the dynamics of $\lambda_{i j}$ does not admit a null solution. Notwithstanding that, the costate equation (57) is indeed irrelevant as the FOC on control $k_{i}$ only contains $\lambda_{i i}$, therefore the set of partial derivatives to be integrated reduces to $2 n$. Since the Hamiltonians are additively separable, Proposition 6 applies and the following Hamiltonian potential can be constructed:

$$
\begin{aligned}
& H_{P}\left(\mathbf{k}, \mathbf{q}, \boldsymbol{\lambda}_{i i}\right)=\sum_{i=1}^{2}\left(\int \frac{\partial H_{i}}{\partial k_{i}} d k_{i}+\int \frac{\partial H_{i}}{\partial q_{i}} d q_{i}\right) \\
= & p_{1} q_{1}+p_{2} q_{2}-c\left(k_{1}^{2}+k_{2}^{2}\right)+\lambda_{11}\left(k_{1}-\varsigma q_{1}\right)+\lambda_{22}\left(k_{2}-\varsigma q_{2}\right) \\
= & \widehat{P}(\cdot)+\lambda_{11} \dot{q}_{11}+\lambda_{22} \dot{q}_{22}
\end{aligned}
$$

with

$$
\widehat{P}(\cdot)=p_{1} q_{1}+p_{2} q_{2}-c\left(k_{1}^{2}+k_{2}^{2}\right) .
$$

\section{Concluding remarks and extensions}

Here we have started developing the analysis of potential functions for differential games, studying a specific class of open-loop games that can be taken as a point of departure for further developments. In particular, we have analysed open-loop games with separate state dynamics. This clearly leaves an open question as to the possibility of proving the existence of potential functions for more general classes of games, and, specifically, in the feedback formulation. One additional open question is whether a Hamiltonian potential can be found to exist in setups where the structure is different from the one we have considered here, e.g. where there is a single state variable for all players. These issus are left for future research. 


\section{References}

[1] Cellini, R. and L. Lambertini (2005), "R\&D Incentives and Market Structure: A Dynamic Analysis", Journal of Optimization Theory and Applications, 126, 85-96.

[2] d'Aspremont, C. and A. Jacquemin (1988), "Cooperative and Noncooperative R\&D in Duopoly with Spillovers", American Economic Review, 78, 1133-37.

[3] Feichtinger, G. (1983), "The Nash Solution of an Advertising Differential Game: Generalization of a Model by Leitmann and Schmitendorf", IEEE Transactions on Automatic Control, 28, 1044-48.

[4] Feichtinger, G., R.F. Hartl and P.S. Sethi (1994), "Dynamic Optimal Control Models in Advertising: Recent Developments", Management Science, 40, 195-226.

[5] Jørgensen, S. and G. Zaccour (2004), Differential Games in Marketing, Dordrecht, Kluwer.

[6] Kamien, M.I. and I. Zang (2000), "Meet Me Halfway: Research Joint Ventures and Absorptive Capacity", International Journal of Industrial Organization, 18, 9951012.

[7] Lambertini, L. and R. Rovelli (2004), "Independent or Coordinated? Monetary and Fiscal Policy in EMU", in R. Beetsma et al. (eds), Monetary Policy, Fiscal Policies and Labour Markets. Macroeconomic Policy Making in the EMU, Cambridge, Cambridge University Press.

[8] Leitmann, G. and W.E. Schmitendorf (1978), "Profit Maximization through Advertising: A Nonzero Sum Differential Game Approach", IEEE Transactions on Automatic Control, 23, 646-50.

[9] Monderer, D. and L.S. Shapley (1996), "Potential Games", Games and Economic Behavior, 14, 124-43.

[10] Slade, M.E. (1994), "What Does an Oligopoly Maximize?", Journal of Industrial Economics, 42, 45-61. 